\title{
METAFÍSICA DEL SER Y DE LA VIDA EN SANTO TOMÁS
}

\author{
Francesc Torralba Roselló \\ Universidad de Barcelona
}

En este trabajo vamos a estudiar el significado del término «ser» y del término «vida» en los textos de Santo Tomás de Aquino. Vamos a tratar de descifrar el contenido semántico de estos vocablos y poner de relieve la relación significativa entre ambos. ¿Qué es el ser? ¿Qué es la vida? ¿Qué vínculo existe entre ser y vida? ¿Qué es primero desde el punto de vista ontológico? ¿Qué es más perfecto? Estas son algunas de las cuestiones que planteamos en el presente artículo.

Los términos «ser» $\mathrm{y}$ «vida» forman parte de ese conjunto de vocablos polisémicos que vertebran el discurso filosófico de Occidente. A lo largo de la historia de la metafísica desde Parménides a Heidegger observamos la presencia de la palabra «ser» en los diferentes discursos especulativos y ontológicos, ya en la antigüedad, ${ }^{1}$ posteriormente en el medievo ${ }^{2}$ y finalmente en la modernidad. ${ }^{3}$ Lo mismo ocurre con el vocablo «vida» aunque no con el mismo protagonismo. ${ }^{4}$

1 Sobre el problema metafísico del ser en la filosofía antigua véanse los siguientes estudios: Aubenque, P., Le problème de l' être chez Aristote, París, PUF, 1977; Coreth, E., Metafísica. Una fundamentación metódicosistemática, Ariel, Barcelona, 1964; De Vries, J., Pensar y ser, Razón y Fe, Madrid, 1953; Fabro, C., Dell' essere di Aristotele allo "esse» di Tommaso: Melanges Gilson (Toronto-París, 1959), pp. 227-247; Finance, J., Conocimiento del ser. Tratado de ontología, Madrid, Gredos, 1971; Gilson, E., El ser y los filósofos, Eunsa, 1978; González, A. L., Ser y participación, Pamplona, Eunsa, 1979; Maritain, J., Siete lecciones sobre el ser y los primeros principios de la razón especulativa, Buenos Aires, Desclée, 1943; Philippe, MD., L'être. Recherche d'une philosophie première, París, Beauchesne, 1970; Raeymaeker, L., Filosofía del ser. Ensayo de síntesis metafisica, Madrid, Gredos, 1968.

2 Sobre el problema del ser en la filosofía medieval véanse los siguientes estudios: Fabro, C., La nozione metafisica di partecipazione secondo S. A. d'Aquino, Milán, 1950; Idem., Actualité et originalité de "essere» homiste: Rev. Thom., 56 (1956), 240-270; 480-507; Idem., Partecipazione e causalitá, Torino, 1960; Idem., Un 
La tarea del filósofo es pensar la realidad, pensar el sentido de lo real. Observa los entes que le rodean y trata de hallar la clave metafísica que le permita conocer la realidad en lo más íntimo, en lo más profundo. La pregunta del filósofo es radical y va dirigida al núcleo de la realidad: ¿Qué son las cosas? ¿Por qué son? ¿Para qué son?

Cautivado por la admiración, se ve impelido a pensar la realidad. Y pensar significa ordenar y someter el conjunto de las percepciones al criterio racional. Observa el filósofo que las cosas son, esto es, que estan presentes en la realidad. Todos los entes tienen algo en común, a saber, que son, es decir, que están ahí, en el mundo. Esta es la primera afirmación metafísica que subsume la pluralidad de los entes bajo una proposición universalmente válida. El árbol es, la rosa es, el papel es, y don Antonio es. Todos estos entes tienen algo en común, tiene el ser (esse est commune omnibus). ${ }^{5}$

Los entes son (primera afirmación), pero no son de la misma manera (segunda afirmación). El árbol no es igual a la rosa, y la rosa no es igual a la piedra y la piedra no es igual a Don Antonio. Todos son, pero según su modo de ser propio, según su esencia.

itineraire de St. Tomas: l'établissement de la distinction reélle entre essence et existence: Rev. Phil, 39, (1939), 285-310; Finance, J., Etre et agir dans la philosophie de Saint Thomas, Roma, U. Gregoriana, 1960; Forment, E., Ser y persona, Barcelona, PPU, 1982; Idem., El «esse» en Santo Tomás: Espíritu, XXXII, (1983), pp. 105. 127; Idem., Introducción a la metafisica, PPU, Barcelona, 1984; Idem, Filosofía del ser, PPU, Barcelona, 1988; Mondin, B., La filosofia dell' essere di S. Tommaso d'Aquino, Roma, Herder, 1964; Canals Vidal, F., Cuestiones de fundamentación, Barcelona, PPu, 1981; Idem., La esencia del conocimiento, Barcelona, PPU, 1987.

3 La mayoría de los estudios sobre la problemática del ser en la Modernidad se centran fundamentalmente en la obra de Heidegger, lo cual es lógico porque en el pensamiento moderno de Descartes a Heidegger la pregunta por el ser queda relegada en un segundo plano, queda olvidada. Heidegger lleva a cabo el giro copernicano de la filosofía del sujeto a la filosofía del ser. Sobre la interpretación del ser de Heidegger hay varios estudios calificados: Corvez, M., La pensée de l'être chez Martin Heidegger; Rev. Thom., 1966; Couturier, F,, Monde et être chez Heidegger, París 1971; Korn, E. A., La question de l'être chez Heidegger: Rev. Thomiste, (1970), pp. 227-263; Berciano, M., La crítica de Heidegger al pensar occidental, Universidad Pontificia de Salamanca, 1990; Idem, La superación de la metafísica en Martín Heidegger, Oviedo, Servicio de Publicaciones de la Universidad, 1992.

4 El término «vida» constituye uno de esos vocablos cuyo significado es plural y está intímamente connectado a un modo de concebir el mundo. Los griegos, con Aristóteles, en primer lugar definieron la vida como automovimiento, los medievales acogieron la definición aristotélica y distinguieron vida de ser. En la modernidad el término vida ha tenido distintas significaciones: como vida biológica en el darwinismo, la vida como experiencia trágica en Nietzsche, la vida como realidad radical en Ortega y Gasset, la vida como impulso y creación en Bergson. .. En el siglo XIX y principios del XX proliferaron las filosofías de la vida, cuyo objeto de especulación metafísica era la vida entendida como fundamento y razón última de la existencia del mundo. El concepto de «vida» no sólo adquiere un papel trascendental en algunas filosofías sino que tiene mucha más amplitud, pues está presente en toda manifestación cultural, artística o religiosa. Así lo recuerda $F$. Canals en su magistral libro Sobre la esencia del conocimiento, Barcelona, PPU, 1987, pág. 584-585: «En efecto, el término vida, en el lenguaje de las Escrituras, en el de la filosofía, en el de la política y en el de la poesía y el teatro, se presenta siempre con un carácter central, que abarca desde la caracterización de la eternidad de Dios, o de la «vida eterna» esperada por los hombres, hasta integrar, como pertenecientes a la vida, la felicidad humana y el infortunio, la actividad teoréctica, la acción moral y las tareas domésticas, políticas de los hombres en la colectividad social, así como la moralidad humana y la actividad creativa de la técnica y del arte».

5. Santo Tomás de Aquino, Suma Teológica, I q. 4 a. 3. 
El ser, por lo tanto, no se dice del mismo modo, y en este sentido no es unívoco, sin embargo, tampoco es equívoco, puesto que todos los entes tienen, de alguna manera, ser. En consecuencia, el ser se dice de un modo analógico. Hay entes que participan más plenamente del ser, y otros que lo tienen levemente. En cualquier caso, lo que resulta evidente es que todos los entes son, pero no son del mismo modo.

Santo Tomás pensó la realidad, y trató de describirla metafísicamente, esto es, a partir de unos principios universalmente válidos y aplicables a todo ente, a toda realidad. Por ello, fundamentó toda su metafísica en el ser, porque el ser es aquello que tienen todos los entes, aunque en diferente grado. Dice en la Suma Teológica: «El ser es lo más íntimo de cada cosa y lo que más profundamente las penetra, ya que, según hemos visto, es principio formal de cuanto en ellas hay». ${ }^{6}$

El ser es lo más profundo del ente (esse est quod profundius omnibus inest), es el fundamento del ente, aquello que le mantiene en la realidad, aquello por lo que existe, es el acto de ser. El ser posibilita los demás actos. Sin el ser el ente no estaría presente en la realidad, el ente no sería nada, o como mucho sería una mera posibilidad. El ente para Santo Tomás no es una mera posibilidad de ser, sino aquello que $e s$, que tiene ser (ens est it quod habet esse). ${ }^{7}$ Dice en otro texto de la Suma: «El mismo ser es la más perfecto de todo, pues es comparado a todo como acto. Pues nada tiene actualidad, a no ser en cuanto es; de donde el mismo ser es la actualidad de todas las cosas, y aún de las mismas formas».8

El ser, por lo tanto, es aquello por lo que los entes son. No se identifica con la existencia, es decir, con la presencia de un ente aquí o allí en un determinado tiempo, sino que es el fundamento de su estar ahí, de su existencia, de su presencia en un determinado tiempo y espacio. El ser pues, es diferente de la existencia, es lo que fundamenta al ente existente.

Todas las formas o actualidades del ente son posteriores al ser y se fundamentan en él. Así por ejemplo, se puede decir afirmar que aquel árbol es verde, es alto, es fecundo y otros atributos más. Pero ninguno de estos atributos tienen sentido si antes no se afirma el ser del árbol, y se dice que el árbol es. Pues aunque el árbol fuere muy fecundo, si éste no fuere,no tuviere ser, entonces sus frutos tampoco serían. Todas las formas y las actualidades de los entes se fundamentan en el ser.

El ser es lo primero, lo más profundo de cada ente. Un árbol puede ser más o menos fecundo pero sino tiene el ser no es nada. Por ello dice Santo Tomás que el ser es la perfección de todas las perfecciones . Porque todas las perfecciones del ente dependen en última instancia de la perfección del ser. El ser es en este sentido, el acto de todos los actos, ${ }^{9}$ la forma de todas las formas, ${ }^{10}$ el principio primero y fundamental del ente.

Así lo expresa también en el De Potencia: «Hay que decir, ante todo que el ser es lo más perfecto, y esto es patente porque el acto es más perfecto siempre que la potencia. Cualquier

6 Idem, S. Th., I q. 8 a. 1

7 Idem, In XII Metaph., lec. 1, n. 2419; In III Sent., d. 6, q. 2, a. 2.

8 Idem, S. Th., I q. 4 a. 3 ad. 3

9 Idem, S. Th., I q. 105 a. $5 \mathrm{c}$

10 Idem, S. Th., I q. 3 a. $3 \mathrm{c}$ 
forma significada no es entendida en acto a no ser que sea puesto el ser. Pues la humanidad o la igneidad puede considerarse como en potencia existente en la materia, o como en el poder del agente, o también como en la inteligencia, pero sólo se dan existentes en acto por el hecho de que tengan ser. De donde hay que decir que el ser es la actualidad de todos los actos, y esto es la perfección de todas las perfecciones». ${ }^{11}$

En relación con esto dice Santo Tomás que el ser no es comparado a otro como el recipiente a lo recibido, sino más bien, como lo recibido al recipiente. ${ }^{12}$ Así pues, cuando se dice el ser del hombre, o el ser del caballo o el ser de cualquier otra cosa, el mismo ser es considerado como algo formal y recibido, no, en cambio, como aquello al que compete el ser.

Santo Tomás mediante este texto trata de aclarar el sentido del ser. El ser debe ser considerado como algo recibido, algo que no pertenece al ente en cuanto tal sino como algo dado, recibido. El ente es, porque participa del ser, porque recibe el ser. En este sentido el ser no debe identificarse con la materia, que es imperfecta y potencialidad pura. El ser no es el recipiente que se llena de perfecciones, como un vaso se llena de agua. El ser es recibido según la esencia de cada ente, según su naturaleza. La piedra recibe al ser de un modo primitivo e imperfecto, la planta lo recibe de un modo más perfecto, el animal todavía sube un escalón en la participación del ser, y el hombre ocupa la cúspide de las sustancias materiales.

En consecuencia el ser no actualidad añadida, o una forma más que se dice del ente, sino la raíz de todas las perfecciones del ente. Sin ella, el ente carecería de ser y sólo existiría en la mente, en la imaginación, como ente pensado.

Los entes son diferentes porque participan de distinto modo del ser. Los hay más complejos, que reciben el ser de un modo más pleno, más fecundo. Otros tan sólo lo tiene de forma incipiente, elemental. Santo Tomás distingue claramente el ser por participación del ser en su plenitud. Aquello que tiene ser y no es el ser, es ente por participación. ${ }^{13}$ ¿Qué significa participar del ser?: «Participar es como coger una parte ;y por ello, cuando algo de modo particular recibe lo que otro pertenece de modo total se dice participar de aquéllo». ${ }^{14}$

Así pues, participar del ser no significa apropiarse de una parte del ser, sino que significa tenerlo sólo en parte, esto es, de un modo imperfecto. El ente no es el ser, sino que participa de él, esto es, lo tiene de forma limitada, imperfecta porque lo recibe según su naturaleza o esencia. La esencia limita la recepción del ser. El ente participa del ser según su propia naturaleza. En Dios la esencia se identifica con el ser, lo que significa que su naturaleza no

11 Idem, S. Th., De Potentia, q. 7 a. 2 ob. 9. Tal como dice E. Forment en Filosofia del ser, Barcelona, PPU, 1988, pág. 122-123: «Al ser no se le puede añadir algo que no esté relacionado con él, pues todo está constituído y tiene realidad por el ser, únicamente puede adicionarsele la nada, que no es. No es posible, por tanto, añadir al ser un elemento más formal, Además, entonces se comportaría como la materia primera con respecto a la forma sustancial, sería potencia en relación a este elemento, el cual lo determinaría como el acto a la potencia, es decir, lo complementaría o perfeccionaría y el ser ya no sería el más perfecto».

12 Cf. S. Th., I q. 4 a. 3.

13 Cf. S. Th., I q. 3 a. 4 ad. 3.

14 Idem, S. Th., I q. 44 a. 1. Sobre el tema de la participación en Santo Tomás es clarificador el libro de E. Forment, Introducción a la metafísica, Barcelona, Eudeba, 1984, pp. 145-148. 
limita en nada al ser, sino que armoniza plenamente con él. Esta identidad sólo se da en Dios, que la suma perfección. En los demás entes, la esencia coharta la plenitud del ser, la naturaleza limita la recepción del ser.

En relación con esto, dice Santo Tomás que las cosas no se distinguen entre sí según que tengan ser, pues, en esto todas convienen. ${ }^{15}$ Las cosas se diferencian porque tienen diversas naturalezas, mediante las cuales reciben el ser de diferentes modos. ${ }^{16}$

En efecto, la diferencia ontológica de los entes no proviene del ser, puesto que todos lo tienen, sino de la esencia de los entes, esto es, de su naturaleza. Cada cual participa del ser según su propia naturaleza. La piedra participa en menor grado del ser que la planta porque su naturaleza recibe el ser en menor grado, esto, de un modo más imperfecto y elemental que la planta.

La tesis tomista de la participación en el ser es omnipresente en toda el corpus metafísico del Doctor Angélico. Mediante la partipación es posible pensar lo real sin sucumbir en el puro equivocismo o en el riguroso univocismo. La realidad es compleja, es plural y multiforme. Sin embargo, también es una, porque el ser está presente en todo ente como su fundamento último. En consecuencia, la realidad es pensable sólo a partir de la tesis de la participación, de otro modo, es imposible el ejercicio del filosofar. El equivocismo niega cualquier tentativa de sistematización de lo real, cualquier cosmovisión ontología de lo que es. El univocismo, por otro lado, niega la realidad de las cosas, que no son iguales, ni idénticas entre sí, sino desiguales y diferentes.

En otro texto de la Suma expone Santo Tomás de nuevo su tesis de la participación: «Es, pues, preciso, que todas las cosas, fuera de Dios, no sean su ser, sino que participen del ser, y por consiguiente, es necesario que todos los entes, que son más o menos perfectos en razón de esta diversa participación, tengan por causa un primer ser que es soberanamente perfecto». ${ }^{17}$

Mediante la tesis de la participación en el ser Santo Tomás descubre la clave metafísica que le permite pensar la multiplicidad sin negar la unidad y la unidad sin pensar la multiplicidad. Todas las cosas son una, en la medida en que todas tienen ser. Sin embargo, todas las cosas son diferentes, y no existen dos cosas iguales en el mundo, porque cada una tiene el ser según su naturaleza. De este modo asume Santo Tomás la dialéctica unidad-multiplicidad heredada de los griegos y lo resuelve mediante este instrumento metafísico que es la participación aplicado al ser.

Dice el Aquinate: «Afirmó Platón que es necesario suponer la unidad antes de toda multiplicidad, y Aristóteles dice que lo que es por excelencia ente y por excelencia verdadero, es la causa de todo ente y de todo lo verdadero, como lo que es sumamente cálido es causa de todo lo cálido». ${ }^{18}$

15 Idem., Suma Contra los Gentiles., Libro I cap. 26. En relación con esto comenta Forment en op. cit, pág. 123: «El «ser» de los distintos entes difiere por las diferentes esencias que lo delimitan de distinta manera, que lo rebajan o lo imperfeccionan en diferente grado».

16 Ibídem.

17 Idem, S. Th., I q. 44 a. 1.

18 Ibídem. 
Santo Tomás aplica este descubrimiento aristotélico en el orden del ser. El ente es actual no por sí mismo sino por el ser. La leña se enciende porque recibe el fuego de un agente exterior que lo actualiza en aquella leña sólo potencial. Por sí misma aquella leña nunca se transformará en fuego, porque le falta el principio de actualización que la transforme. El ente recibe el ser también de un agente exterior, de un agente que debe tenerlo en toda su plenitud. Y este ente cuya esencia se identifica con el ser, Dios, es pura actualidad, perfección sublime que entrega el ser a los entes y de este modo son actuales.

La misma idea hallamos en otro texto de la Suma: «Solamente Dios es su ser, y en todas las demás cosas el ser difiere de la esencia, según hemos dicho, por donde evidentemente se ve que sólo Dios es el ser por esencia y que todas las demás cosas son seres por participación». ${ }^{19}$ $\mathrm{Y}$ añade en otro lugar que el ser participado viene a considerarse como el acto del ser que lo participa. Ahora bien, es preciso que toda forma creada subsistente tenga el ser participado, puesto que incluso «la misma vida» o lo que así quiere llarmarse, «participa del ser», como dice Dioniso. Mas el ser participado está limitado por la capacidad del que lo participa. Por tanto, sólo Dios, que es su mismo ser, es acto puro, infinito... ${ }^{20}$

\section{II}

Los entes participan del ser según su naturaleza. Cuanto más participen del ser más perfectos son pues el ser es la perfección de todas las perfecciones, la actualidad de todos los actos tal y como hemos visto. Existe una gradación, una jerarquía participativa. Hay entes que tienen el ser de un modo más pleno y otro de un modo más elemental. ${ }^{21}$

El hombre común se percata de ello cuando observa la naturaleza. Contempla, primero, la pluralidad de los entes, y posteriormente distingue las distintas cosas que ve según su grado de perfección. Hay entes complejos que se mueven autónomamente, que entienden, que aman, que crean, y otros que permanecen estáticos carentes de dinamismo.

¿Cuál es el criterio de perfección? ¿Cómo ordenar los entes en función de su perfección?. Santo Tomás aclara perfectamente el criterio que se tiene que utilizar para ordenar los entes jerárquicamente: «La perfección de cualquier cosa es proporcionada al ser de la misma: ninguna perfección le vendría, por ejemplo, al hombre por su sabiduría si no fuera sabio por ella, y así los otros entes. Por consiguiente, en una cosa, el modo de su grado en el ser marca el modo de su perfección: porque se dice que una cosa es más perfecta o menos perfecta, según su ser sea determinado a un modo especial de mayor o menor perfección».22

19 Idem, S. Th., I q. 61 a. 1.

20 Cf. S. Th., I q. 75 a. 5 ad. 4.

21 El tema de la escala de los seres está expuesto brillantemente en la obra de J. Bofill, La escala de los seres o el dinamismo de la perfección, Barcelona, Ed. Cristiandad, 1950. Forment trata de esta cuestión en Filosofía del Ser, edit. cit. pp. 119-127.

22 Idem, S. C. G., L. 1 cap. 28. 
El texto es claro y explícito por sí mismo. La perfección de una cosa está en relación directamente proporcional al ser de la misma. Cuanto más ser, más perfecta es la cosa. El modo de su grado en el ser marca el modo de su perfección (secundum modum quo res habet esse, est suus modus in nobilitate). La relación entre ser y perfección es directamente proporcional. En relación con esto añade Santo Tomás que si hay un ser a quien pertenezca toda la virtualidad del ser, no puede carecer de perfección alguna que exista en los demás. ${ }^{23}$ Pero le pertenece al ser con toda virtualidad lo que es su propio ser, de la misma manera que, si existiese una blancura separada no podría carecer de lo que es propio de la blancura, pues a un objeto blanco le falta algo propio de esta cualidad por ser defecto del sujeto receptor, que la recibe según su modo particular y no conforme a toda la potencialidad de la blancura. ${ }^{24}$

En Dios la esencia se identifica con el Ser. Dios no tiene el ser sino que es el Ser, es la fuente de toda actualidad, es el fundamento de todo ente. Si Dios es el Ser en plenitud y la perfección es proporcional al ser, entonces Dios también es máximemente perfecto por tener el ser en su totalidad. Ahora bien, Dios que es su propio Ser, como más arriba se ha probado, posee el ser con toda su virtualidad. Luego no puede carece de ninguna de las perfecciones que convengan a cualquier otro. ${ }^{25}$

Si el ser se relaciona con la perfección, el no-ser, esto es la falta de ser, se relaciona con la imperfección. La esencia limita la recepción del ser, de tal modo que el ente creado no es absolutamente perfecto sino que tiene imperfección motivada por su naturaleza que coharta la actualidad del ser, y por lo tanto limita el grado de perfección.

En Dios todo es perfección porque todo es Ser. En el ente creado la perfección que tiene le viene del ser y la imperfección del modo como participa del ser, modo que viene tutelado por la naturaleza del ente en cuestión. En consecuencia la imperfección del ente, no proviene nunca del ser, sino de la esencia, y si alguna perfección tiene un ente se debe reconocer que aquélla viene del ser y no de la esencia.

Así lo expresa Santo Tomás mucho mejor que nosotros: «Así como la bondad y la perfección adviene a una cosa en cuanto es, así también toda imperfección le adviene en cuanto de alguna manera no es. Pero Dios, así como posee totalmente al ser, del mismo modo carece de todo no ser, porque una cosa se aleja del no ser en la medida en que es. Dios, por lo tanto, carece de imperfección». ${ }^{26} \mathrm{Y}$ añade que aquellos entes que sólo tienen ser, no son imperfectos por imperfección del Ser absoluto, pues no tienen el ser en toda su comprehensión, sino que participan del mismo de un modo particular e imperfectísimo. ${ }^{27}$

En el capítulo sexto del De ente y esencia Santo Tomás ordena los entes según su grado de participación en el ser. En la cúspide de la escala de los entes hallamos a Dios que es el Ser, la suma perfección. Dios es sólo ser (Deus est esse tantum), porque su esencia no es otra que su ser (essentia sua no est aliud quam esse suum) ${ }^{28}$

23 Ibídem.

24 Ibídem.

25 Ibídem.

26 Ibídem.

27 Cf. De ente y esencia, trad. E. Forment, op. cit, p. 197.

28 Ibídem. 
Después de Dios hallamos a las sustancias creadas espirituales en las cuales el ser es otro que la esencia aunque la esencia sea sin materia. ${ }^{29}$ Estas sustancias intelectuales carecen de materia, por lo que su esencia se identifica sólo con la forma. Tienen el ser aunque no plenamente sino participativamente. Su ser no es absoluto, sino recibido (non esta absolutum sed receptum) y por esto limitado y finito a la capacidad de la naturaleza del que lo recibe..$^{30}$

Al final del capítulo Santo Tomás desciende un grado más en las escala de los entes y trata de las sustancias creadas compuestas de materia y forma. Estas reciben el ser y por ello dice Santo Tomás que tienen su ser por otro (ab alio esse habent).

\section{III}

Santo Tomás observa que en el conjunto de los entes creados materiales hay algunos que se mueven por sí mismos y otros estáticos que no tienen la capacidad de autolocomoción. Los entes que se mueven a sí mismos como el la planta, el animal, el hombre, son entes que tienen vida. Así define Santo Tomás la vida: «El nombre de vida se toma de algo que aparece exteriormente en las cosas, y que consiste en que se muevan a sí mismas. Pero no fue impuesto para significar esto, sino para significar la sustancia a la que por naturaleza conviene moverse espontáneamente o de algún modo impulsarse a la operación, y por lo tanto, vivir no es otra cosa que existir en determinada naturaleza, y vida significa esto mismo abstracto como carrera es el mismo correr enunciado en abstracto».32

En efecto, la vida es un modo de ser que compete a las sustancias creadas materiales. Hay entes que se mueven y hay entes que son estáticos. Tanto los que se mueven como los que están en reposo son, participan del ser; pero unos participan del ser de un modo dinámico, y son por ello, entes vivos, criaturas vivas. La vida es el término abstracto que designa este modo concreto de ser, que es el vivir. La piedra es pero no vive porque es estática, el hombre es y su modo de ser es la vida porque es dinámico, autolocomotivo.

La misma idea reitera en otro lugar de la Suma: «Pues bien, lo primero que nos mueve a decir que un animal vive es que empiece a moverse por sí mismo, y pensamos que sigue viviendo mientras notamos en él este movimiento, y cuando ya no se mueve por sí, sino sólo a impulso de otro, decimos que ha muerto por falta de vida». ${ }^{33}$

Y añade a continuación que hablando con propiedad, son vivientes los seres que se mueven a sí mismos, cualquiera que sea la especie de movimiento, bien se tome en sentido estricto

29 Ibídem, p. 207.

30 Ibídem, p. 205

31 Cf. S. Th., I q. 18 a. 2. F. Canals estudia el concepto de vida en los textos de Santo Tomás en la sexta parte de su magna obra Sobre la esencia del conocimiento, Barcelona, PPU, 1987, pp. 579-641. Así comenta la definición tomista el sabio profesor barcelonés: «La vida se patentiza así por el movimiento, y el viviente puede ser definido como el ente natural al que por su naturaleza compete el poder activo de moverse en orden de sus operaciones» (p. 585).

32 Idem, S. Th., I q. 18 a. 1 ad. 3.

33 Ibídem. 
como acto de lo imperfecto, esto es, de lo que está en potencia; bien en sentido amplio, como acto de lo perfecto, sentido en que también se llama movimiento a lo actos de entender y sentir. ${ }^{34}$

Santo Tomás precisa en estos textos el significado del término vida. El viviente es el ente que se mueve a sí mismo, sin embargo es necesario comprender el vocablo «movimiento» en sentido lato, esto es, amplio. El movimiento es el acto de ser en potencia en cuanto tal. ${ }^{35}$ Moverse significa pasar de la potencia al acto. En este sentido es movimiento cualquier traslación, generación, corrupción, es decir, todo cambio cualitativo o cuantitativo. De igual modo, la actividad de entender, o de amar o de desear también debe considerarse movimiento.

Santo Tomás observa que en del conjunto de los seres vivos no todos participan de la vida en el mismo grado. Así por ejemplo, la planta se mueve y por ello vive, pero también se mueve el animal, y cualquier hombre en su sano juicio reconocerá que la vida del animal es distinta de la de la planta. Por lo tanto el concepto de vida en estos entes dinámicos no es unívoco pero tampoco equívoco sino más bien analógico. Todos se mueven a sí mismos, y por ello son vivos, pero el modo de vivir, no es el mismo, sino analógico.

\section{IV}

Santo Tomás lleva a cabo una ordenación de los seres vivos en función de su grado de participación en la vida. De nuevo surge la pregunta que ya nos habíamos formulado en la jerarquía ontológica de los entes: ¿Qué criterio utilizar para separar los diferentes grados de vida? ¿Como podemos afirmar que un ente vive más que otro?

El Aquinate responde magistralmente a estas cuestiones siguiendo la misma lógica que antes mostró al plantearse la la pregunta por el ser: «Si la vida se atribuye a los entes que obran por sí mismos y no movidos por otros, cuanto con mayor perfección convenga esto a un ente, tanto más perfecta será la vida que hay en él». ${ }^{36}$

La perfección en los entes vivos se gradua por el modo en que se mueven. Cuánto más autónomo sea un ser, cuánto menos dependa su movimiento del movimiento de otro, más perfecto será.El ser más vivo es el que con más independencia se mueve a sí mismo, es el que no está sujeto al impulso de ningún otro ente.

En base a este criterio, Santo Tomás constituye la siguiente jerarquía: «Cuatro son los géneros de vivientes que hay en este mundo, de entre los cuales, unos sólo son aptos para el

34 Idem, S. Th., I q. 9 a. 1 c.

35 Santo Tomás trata de la esencia del movimiento en distintos lugares de la Suma. Véase: I q. 9 a. 1 ad1; q. 14 a. 1 ad. 2 ; q. 18 a. 1 c; a. 3 ad. $1 ;$ q. 19 a. 1 ad. $1 ;$ q. 53 a. 1 ad. $2 ;$ q. 58 a. 1 ad. $1 ;. .$. A. Prevosti ha estudiado ámpliamente el tema del movimiento en Aristóteles y en Santo Tomás en La física d'Aristòtil, Barcelona, PPU, 1984, pp. 295-324.

36 Idem, S. Th., I q. 18 a. 3. Véase para los grados de vida: Forment, E., Introducción a la metafísica, Barcelona, Eudeba, 1984, pp. 160-165. 
nutrirse y para lo que de ello se deriva: el desarrollo y la reproducción;otros alcanzan, además a sentir, como los animales inmóviles, por ejemplo las ostras; otros llegan al movimiento local, como los animales perfectos, por ejemplo los cuadrúpedos, las aves...; y otros, por fin, alcanzan a entender, como el hombre».37

Esta clasificación se realiza en función del grado de autonomía. Atendiendo a esta independencia observamos estos cuatro grados de vida. En cada grado de vida Santo Tomás distingue tres cosas: el fin de las operaciones de los vivientes, la forma por la que obran, y la ejecución de la acción. El Aquinate justifica su jerarquía en base a esta triple. Veamos la argumentación desarrollada.

El primer grado de vida lo ocupan los vegetales. Estos se mueven a sí mismos pero no hacia un fin o una forma determinada sino que ésto viene prefijado por la naturaleza. ${ }^{38}$

En el segundo grado están los animales que gozan de más autonomía. Son entes que se mueven no sólo en orden a la ejecución del movimiento, sino además para adquirir la forma que le da origen. $Y$ de esta clase son los animales, que tienen como principio de su movimiento una forma, no aneja a la naturaleza sino adquirida por sus sentidos; por lo cual cuanto más perfectos sean éstos, con tanta mayor soltura se mueven... no son ellos, los que se determinan a sí propios el fin de sus actos o movimientos, sino que esto lo llevan anejo a su naturaleza, cuyo instinto los inclina a hacer lo que hacen movidos por la forma que perciben sus sentidos..$^{39}$

El tercer grado de vida pertenece al hombre. En él se da más autonomía que en el animal, aunque no absoluta. Por encima de estos animales están los entes que se mueven también en orden a un fin que ellos mismos se fijan, cosa imposible de hacer si no es por medio de la razón y del entendimiento, al que corresponde conocer la relación que hay entre el fin y lo que a su logro conduce y subordinar ésto a aquéllo. El modo más perfecto de vivir es el de lo entes dotados de entendimiento que son a su vez, los que con mayor perfección se mueven a sí mismos. ${ }^{40}$

El hombre es la criatura que goza de más autonomía en el mundo puesto que su vida se dirige a un fin que no viene determinado por la naturaleza sino por su propia razón y entendimiento. Aun así y a pesar de ser el ente más perfecto del mundo, reconce Santo Tomás que hay en él movimientos que no dependen de su juicio particular sino que vienen impuestos por la naturaleza.

Así lo expresa Santo Tomás: «Aunque nuestro entendimiento tenga la iniciativa en orden a conseguir algunas cosas, otras hay, sin embargo, que de antemano le impone la naturaleza, como son los primeros principios, acerca de los cuales no puede cambiar de parecer, y el último fin, que no puede por menos de querer $y$, por consiguiente, si en orden a algunas cosas se mueve a sí mismo, respecto de otras es necesario que sea movido». ${ }^{41}$

37 Idem, S. Th., I q. 18 a. 2.

38 Idem, a. 3.

39 Ibídem.

40 Ibídem.

41 Ibídem. 
En consecuencia, la vida del hombre no es radicalmente autónoma porque está sujeta a unas leyes de la naturaleza, a unos principios primeros que no pueden moverse y además, tiende inevitablemente, por su propia constitución metafísica a un fin último, que es Dios, fuente de su ser, bálsamo de su vida.

La perfección del viviente está íntimamente relacionada con la intelectualidad del mismo. Cuánto más intelectual más autónomo, más independiente de las directrices de la naturaleza. El hombre vive más que el animal porque goza de vida intelectual y puede pensar el fin y los medios necesarios para alcanzarlo. Aun así, hemos visto que en el hombre la vida no es absolutamente autónomo porque depende de unos principios primeros que le son extrínsecos. Dice el Santo en la Suma contra los gentiles que el supremo grado de vida es el que es según el entendimiento, pues el entendimiento reflexiona sobre sí mismo, y puede entenderse a sí mismo. Pero también en la vida intelectual se hallan grados diversos. Pues el entendimiento humano, aunque pueda conocerse a sí mismo, toma sin embargo el primer comienzo de su conocimiento de algo extrínseco: porque en él no hay entender sin imagen. ${ }^{42}$

En la sustancias intelectuales la autonomía tampoco es completa porque en ellos no es lo mismo el enteder y el ser, lo que significa que algo de su ser no pertenece al entender y por lo tanto no es su propia sustancia. El ser verdaderamente autónomo es aquel en donde se da la identidad entre el ser y el entender, donde su vida en sentido extensivo es igual a su entendimiento. Este ser totalmente autónomo que no depende de ningún principio extrínseco, este ser que se identifica plenamente con la vida, que él es la vida, es Dios. Con la identificación del entender y el ser, se llega al último grado de perfección en la vida, se llega a la vida perfecta en la que se da una completa autonomía. ${ }^{43}$

Por lo tanto, en la escala de la vida, como en la escala del ser, Dios ocupa la cúspide. En Dios la esencia se identifica con el ser y el ser se identifica con el entender, por lo cual es lógico afirmar que la esencia de Dios es entender. En Dios se da la vida en sentido pleno porque su esencia que es el ser se identifica con el entender. ${ }^{44}$ El ejercicio de entender en Dios es completamente autónomo y no depende de ningún principio extrínseco, como ocurría en el caso del hombre.

\section{V}

La escala del ser y la escala de la vida no son dos gradaciones autónomas y menos aún paralelas sino que están íntimamente connectadas, de tal modo que la escala de la vida se edifica sobre la escala del ser.

Hay entes que son, pero no viven porque no se mueven a sí mismos, y hay entes que son y su modo de ser es dinámico, esto es, participan de la vida. Sin embargo estos entes no vivirían

42 S. C. G., Libro IV cap. 11.

43 Ibídem.

44 Idem, S. Th., I q. 18 a. 3. 
si con anterioridad no participaran del ser. Su modo de participar en el ser es más perfecto, es más autónomo que en los entes estáticos, y en esto se distinguen de aquéllos. Si la vida fuera antes que el ser, entonces los entes estáticos, no serían nada, pues no se mueven a sí mismos. Resulta obvio afirmar que el ser es anterior a la vida,y que la vida no es algo que se añade al ente sino que se constituye como participación en el ser. El vivir es en sí mismo un nivel de perfección en el ser, lo que significa que el viviente participa más perfectamente que el no viviente del ser. En consecuencia, la escala de la vida pertenece a la escala del ser. El viviente es viviente porque participa de un modo más pleno del ser. Esta participación más plena le confiere dinamismo, autolocomoción. La vida del viviente es fruto de su participación en el ser.

El ente inmóvil también participa del ser, pero en menor grado. Si participar del ser más plenamente entonces alcanzaría el automovimiento y cierta autonomía. La vida, por lo tanto, indica un grado de participación en el ser que se caracteriza por su dinamicidad. Ahora bien, este carácter móvil, no es unívoco sino analógico, cuanto más participe el ente del ser, más dinámico, más autónomo, más perfecto en su constitución metafísica. Como dice Santo Tomás en la Suma contra los gentiles el vivir de los vivientes es su mismo ser (Vivere autem viventium est ipsum esse)..$^{45}$

La vida no es anterior al ser, ni un principio independiente, sino que indica un modo de participar en el ser característico de los entes que se mueven a sí mismo. Para los vivientes su ser es el vivir, y su vivir es el ser. Lo que define su grado de participación en el ser es su vida y los distingue de los que solamente son y no viven porque su grado de participación es muy elemental. Aun así, estos entes gozan de la perfección del ser, y de este modo son actuales y no meramente posibles, su esencia cobra realidad y se hacen presentes.

45 Idem, S. Th., I q. 4 a. 2. 